\title{
ON A CONJECTURE OF HARRIS
}

\author{
ANANYO DAN
}

\begin{abstract}
For $d \geq 4$, the Noether-Lefschetz locus $\mathrm{NL}_{d}$ parametrizes smooth, degree $d$ surfaces in $\mathbb{P}^{3}$ with Picard number at least 2. A conjecture of Harris states that there are only finitely many irreducible components of the Noether-Lefschetz locus of non-maximal codimension. Voisin showed that the conjecture is false for sufficiently large $d$, but is true for $d \leq 5$. She also showed that for $d=6,7$, there are finitely many reduced, irreducible components of $\mathrm{NL}_{d}$ of non-maximal codimension. In this article, we prove that for any $d \geq 6$, there are infinitely many non-reduced irreducible components of $\mathrm{NL}_{d}$ of non-maximal codimension.
\end{abstract}

\section{INTRODUCTION}

The underlying field is $\mathbb{C}$. By surface we will always mean a projective surface in $\mathbb{P}^{3}$. A classical result in the theory of surfaces, stated by M. Noether and later proved by Lefschetz, says that for any $d \geq 4$, a very general, smooth, degree $d$ surface in $\mathbb{P}^{3}$ is of Picard number 1 (by Picard number we mean the rank of the Néron-Severi group). Here, very general means that the points on the parametrizing space $\mathbb{P}\left(H^{0}\left(\mathbb{P}^{3}, \mathcal{O}_{\mathbb{P}^{3}}(\mathrm{~d})\right)\right)$ of degree $d$ surfaces in $\mathbb{P}^{3}$, corresponding to such surfaces, lie outside a countable union of proper, closed subsets of $\mathbb{P}\left(H^{0}\left(\mathbb{P}^{3}, \mathcal{O}_{\mathbb{P}^{3}}(\mathrm{~d})\right)\right)$. The Noether-Lefschetz locus, denoted $\mathrm{NL}_{d}$, is then defined to be the locus of smooth, degree $d$ surfaces in $\mathbb{P}^{3}$ with Picard number at least 2 . The irreducible components of the Noether-Lefschetz locus have a natural (analytic) scheme structure, which we will now describe.

Denote by $U_{d} \subseteq \mathbb{P}\left(H^{0}\left(\mathbb{P}^{3}, \mathcal{O}_{\mathbb{P}^{3}}(\mathrm{~d})\right)\right)$ the open subscheme parametrizing smooth projective hypersurfaces in $\mathbb{P}^{3}$ of degree $d$. Let

$$
\pi: \mathcal{X} \rightarrow U_{d}
$$

be the corresponding universal family. For a given $u \in U_{d}$, denote by $\mathcal{X}_{u}:=\pi^{-1}(u)$. Denote by

$$
\mathbb{H}:=R^{2} \pi_{*} \mathbb{Z} \text { and } \mathcal{H}:=R^{2} \pi_{*} \mathbb{Z} \otimes \mathcal{O}_{U_{d}} .
$$

Using the Ehresmann's theorem, it is easy to check that $\mathbb{H}$ is a local system, hence $\mathcal{H}$ is a vector bundle. Fix a point $o \in \mathrm{NL}_{d}$ and $U \subseteq U_{d}$ a simply connected open neighbourhood of $o$ in $U_{d}$ (under the analytic topology). It is easy to check that the restriction of $\mathbb{H}$ to $U$ is trivial and any class $\gamma_{0} \in H^{2}\left(\mathcal{X}_{o}, \mathbb{Z}\right)$ defines by flat transform, a section $\gamma \in \Gamma(U, \mathbb{H})$. Let $\bar{\gamma}$ be the image of $\gamma$ in $\mathcal{H} / F^{1} \mathcal{H}$, where $F^{1} \mathcal{H} \subset \mathcal{H}$ is a vector subbundle such that for every $u \in U$, the fiber $\left(F^{1} \mathcal{H}\right)_{u} \subset \mathcal{H}_{u}$ can be identified with $F^{1} H^{2}\left(\mathcal{X}_{u}, \mathbb{C}\right) \subset H^{2}\left(\mathcal{X}_{u}, \mathbb{C}\right)$ (see $\left.[12, \S 10.2 .1]\right)$. If $\gamma_{0}$ belongs to $H^{1,1}\left(\mathcal{X}_{o}, \mathbb{C}\right)$ i.e., $\gamma_{0}$ is a Hodge class, then the Hodge locus associated to $\gamma_{0}$, denoted $\operatorname{NL}\left(\gamma_{0}\right)$, is defined as

$$
\operatorname{NL}\left(\gamma_{0}\right):=\{u \in U \mid \bar{\gamma}(u)=0\},
$$

where $\bar{\gamma}(u)$ denotes the value at $u$ of the section $\bar{\gamma}$. The Hodge locus is equipped with a natural scheme structure (see $[13, \S 5.3 .1]$ ). The intersection of $\mathrm{NL}_{d}$ with $U$ is the union of $\mathrm{NL}\left(\gamma_{0}\right)$ as $\gamma_{0}$ ranges over the Hodge classes of $\mathcal{X}_{u}$ for all $u \in U$, such that the Hodge class is not a multiple of $c_{1}\left(\mathcal{O}_{\mathcal{X}_{u}}(1)\right)$ (see $\left.[13, \S 5.3 .3]\right)$. We say that the closure $\overline{\mathrm{NL}\left(\gamma_{0}\right)}$ of $\mathrm{NL}\left(\gamma_{0}\right)$ (in the Zariski topology), is an irreducible component of $\mathrm{NL}_{d}$, if the underlying topological space is irreducible and as an

Date: March 3, 2020.

2010 Mathematics Subject Classification. 14D07, 14C05, 14C20.

Key words and phrases. Harris conjecture, Noether-Lefschetz locus, Hodge locus, flag Hilbert schemes. 
(analytic) scheme is not contained properly in any irreducible component of $\overline{\mathrm{NL}\left(\gamma^{\prime}\right)}$ for some Hodge class $\gamma^{\prime}$ over $\mathcal{X}_{o}$, where $\gamma^{\prime}$ is not a multiple of $c_{1}\left(\mathcal{O}_{\mathcal{X}_{o}}(1)\right)$. Two irreducible components of $\mathrm{NL}_{d}$ are isomorphic if they are isomorphic as analytic schemes (scheme structure as Hodge loci). It was shown by Ciliberto-Harris-Miranda [1] that any irreducible component $L$ of $\mathrm{NL}_{d}$ satisfies the inequality:

$$
d-3 \leq \operatorname{codim}\left(L, U_{d}\right) \leq\left(\begin{array}{c}
d-1 \\
3
\end{array}\right)
$$

If $\operatorname{codim}\left(L, U_{d}\right)=\left(\begin{array}{c}d-1 \\ 3\end{array}\right)$, then $L$ is called a general component. Otherwise, $L$ is called a special component. Harris conjectures the following on the special components of the Noether-Lefschetz locus (see $[2,11])$ :

Conjecture (Harris). Fix an integer $d \geq 5$. Then,

(1) Topological Harris conjecture: Ignoring the natural analytic scheme structure (as Hodge locus) on the irreducible components of $\mathrm{NL}_{d}$, there are finitely many topological, special components of $\mathrm{NL}_{d}$.

(2) Analytic Harris conjecture: The Noether-Lefschetz locus $\mathrm{NL}_{d}$ contains finitely many special, irreducible components (by irreducible component we mean as above).

For $d \leq 5$ the conjectures hold true (see [9, Theorem 0.2]). The conjectures have been shown to be false by Voisin [11] for sufficiently large $d$. For $d=6,7$, Voisin proved in [10] that $\mathrm{NL}_{d}$ has finitely many reduced, special components. But there are several questions that are still open: What is the largest $d^{\prime}$ such that the Harris conjectures hold true for all $d \leq d^{\prime}$ ? For those $d$ for which the Harris conjectures fail, what is the largest $k$ such that there are finitely many special components of codimension at most $k$ and infinitely many special components of codimension strictly greater than $k$ ? In this article we give a complete answer to these questions for the analytic Harris conjecture. We show:

Theorem 1.1 (see Theorem 2.4). Let $d \geq 6$ and $X$ be a general smooth degree $d$ surface containing two coplanar lines, say $L_{1}, L_{2}$. Denote by $\gamma_{a}:=\left[L_{1}\right]+a\left[L_{2}\right] \in H^{2}(X, \mathbb{Z}) \cap H^{1,1}(X, \mathbb{C})$, for any $a \in \mathbb{Z}$. Then, for any $a, b \geq 0$ with $a \neq b$, we have $\operatorname{NL}\left(\gamma_{a}\right) \neq \operatorname{NL}\left(\gamma_{b}\right)$. Moreover,

$$
\operatorname{codim} \mathrm{NL}\left(\gamma_{0}\right)=d-3, \operatorname{codim} \mathrm{NL}\left(\gamma_{1}\right)=2 d-7 \text { and } \operatorname{codim} \mathrm{NL}\left(\gamma_{a}\right)=2 d-6 \text { for } a \geq 2 \text {. }
$$

In particular, we have following table which gives the number of irreducible components of $\mathrm{NL}_{d}$ with the given codimension:

\begin{tabular}{|c|c|}
\hline codimension & number of irr. components \\
\hline$<d-3$ & 0 \\
$\mathrm{~d}-3$ & 1 \\
$2 \mathrm{~d}-7$ & 1 \\
$\leq 2 d-7$ & 2 \\
$2 \mathrm{~d}-6$ & $\infty$ \\
\hline
\end{tabular}

The theorem immediately disproves the analytic Harris conjecture for $d \geq 6$. Recall, Voisin in [11] uses the existence of infinitely many general components of $\mathrm{NL}_{4}$ to produce infinitely many sufficiently high degree special components (after replacing the coordinates with general high degree polynomials). In particular, the topological space underlying the special components are distinct. Her counterexample relies on a numerical inequality that holds only for sufficiently large $d$ (see [11, p. 686]). In contrast, we simply study the scheme structure of the Hodge locus corresponding to different linear combinations of coplanar lines. In particular, we show that the space parametrizing smooth, degree $d$ surfaces containing 2 coplanar lines can be equipped with infinitely many (distinct) scheme structures naturally arising as the Hodge loci associated to different combinations of the two coplanar lines (see Theorem 2.4). As a result, the infinite 
number of special components in this article have the same underlying topological space (but different analytic scheme structures), thus giving us an entirely different set of counterexamples from those in [11]. The topological Harris conjecture is still open for small values of $d$. In a recent preprint, Movasati in [4] uses computer calculations for explicit values of $d$ to give a description of possible counterexamples to the topological Harris conjecture.

Acknowledgements: The author is currently supported by ERCEA Consolidator Grant 615655NMST, by the Basque Government through the BERC 2018 - 2021 program and Gobierno Vasco Grant IT1094 - 16, by the Spanish Ministry of Science, Innovation and Universities: BCAM Severo Ochoa accreditation SEV-2017 - 0718.

\section{PRoOf of MAIN THEOREM}

In this section we prove Theorem 1.1. Fix an integer $d \geq 6$.

2.1. Cohomology computations of the invertible sheaves associated to lines. To prove Theorem 1.1 we need the following basic computation on the first and second cohomology group of the invertible sheaf associated to a line contained in a smooth, degree $d$ surface in $\mathbb{P}^{3}$.

Lemma 2.1. Let $X$ be a smooth, degree $d$ surface containing two coplanar lines, say $L, L^{\prime}$. Then,

$$
H^{0}\left(\mathcal{O}_{X}\left(L^{\prime}\right)\right)=H^{0}\left(\mathcal{O}_{X}(L)\right)=\mathbb{C}=H^{0}\left(\mathcal{O}_{X}\left(L \cup L^{\prime}\right)\right) \text { and } H^{1}\left(\mathcal{O}_{X}(L)\right)=0=H^{1}\left(\mathcal{O}_{X}\left(L^{\prime}\right)\right) .
$$

Proof. By the adjunction formula, $L^{2}=-2-(d-4)=2-d$, which is less than zero for $d \geq 6$. Hence, $H^{0}\left(\mathcal{N}_{L \mid X}\right)=0$. Using the short exact sequence

$$
0 \rightarrow \mathcal{O}_{X} \rightarrow \mathcal{O}_{X}(L) \rightarrow \mathcal{N}_{L \mid X} \rightarrow 0
$$

we conclude that $\mathbb{C}=H^{0}\left(\mathcal{O}_{X}\right)=H^{0}\left(\mathcal{O}_{X}(L)\right)$. Similarly, we can show that $\mathbb{C}=H^{0}\left(\mathcal{O}_{X}\left(L^{\prime}\right)\right)$. Next, we consider the short exact sequence:

$$
0 \rightarrow \mathcal{O}_{X}(L) \rightarrow \mathcal{O}_{X}\left(L \cup L^{\prime}\right) \rightarrow \mathcal{O}_{L^{\prime}} \otimes \mathcal{O}_{X}\left(L \cup L^{\prime}\right) \rightarrow 0
$$

obtained by tensoring with $\mathcal{O}_{X}\left(L \cup L^{\prime}\right)$ the short exact sequence:

$$
0 \rightarrow \mathcal{O}_{X}\left(-L^{\prime}\right) \rightarrow \mathcal{O}_{X} \rightarrow \mathcal{O}_{L^{\prime}} \rightarrow 0 .
$$

Note that, $L^{\prime} .\left(L+L^{\prime}\right)=1+(2-d)<0$ for $d \geq 6$. This implies, $H^{0}\left(\mathcal{O}_{L^{\prime}} \otimes \mathcal{O}_{X}\left(L \cup L^{\prime}\right)\right)=0$. By (2.2), we then have $H^{0}\left(\mathcal{O}_{X}\left(L \cup L^{\prime}\right)\right)=H^{0}\left(\mathcal{O}_{X}(L)\right)=\mathbb{C}$. This proves the first part of the lemma. We now show that $H^{1}\left(\mathcal{O}_{X}(L)\right)=0=H^{1}\left(\mathcal{O}_{X}\left(L^{\prime}\right)\right)$.

Recall, the Castelnuovo-Mumford regularity of $\mathcal{I}_{L}$ is one (see [6, Example 1.8.2]). This implies that $H^{i}\left(\mathcal{I}_{L}(j)\right)=0$ for $i \geq 1$ and $j \geq 0$. Consider now the short exact sequence:

$$
0 \rightarrow \mathcal{I}_{X}(d-4) \rightarrow \mathcal{I}_{L}(d-4) \rightarrow \mathcal{O}_{X}(-L)(d-4) \rightarrow 0 .
$$

As $\mathcal{I}_{X} \cong \mathcal{O}_{\mathbb{P}^{3}}(-d)$, we have $H^{2}\left(\mathcal{I}_{X}(d-4)\right)=0$. Using the long exact sequence associated to (2.3), we conclude that $H^{1}\left(\mathcal{O}_{X}(-L)(d-4)\right)=0$. By Serre duality this implies

$$
H^{1}\left(\mathcal{O}_{X}(L)\right)=H^{1}\left(\mathcal{O}_{X}(-L)(d-4)\right)=0 .
$$

Similarly, we can show that $H^{1}\left(\mathcal{O}_{X}\left(L^{\prime}\right)\right)=0$. This proves the lemma. 
2.2. Flag Hilbert schemes. Let $X$ be a smooth, degree $d$ surface in $\mathbb{P}^{3}$ containing two (distinct) coplanar lines, say $L_{1}$ and $L_{2}$. Denote by $P_{0}$ (resp. $P_{1}, Q_{d}$ ) the Hilbert polynomial of $L_{1}$ (resp. $L_{1} \cup L_{2}, X$ ). Denote by $\operatorname{Hilb}_{P_{0}, P_{1}, Q_{d}}$ the flag Hilbert scheme parametrizing triples $\left(Z_{1} \subset Z_{2} \subset Z_{3}\right.$ ) with $Z_{1}$ (resp. $Z_{2}, Z_{3}$ ) having Hilbert polynomial $P_{0}$ (resp. $P_{1}, Q_{d}$ ). Denote by Hilb $P_{i}$ and Hilb $_{Q_{d}}$ the Hilbert scheme associated to the Hilbert polynomial $P_{i}$ and $Q_{d}$, respectively for $i=0,1$. See $[8, \S 4.3,4.5]$ for a detailed discussion on (flag) Hilbert schemes.

Proposition 2.2. The flag Hilbert scheme $\operatorname{Hilb}_{P_{0}, P_{1}, Q_{d}}$ is reduced. In particular, the schemetheoretic image under the natural projection map

$$
\text { pr : } \operatorname{Hilb}_{P_{0}, P_{1}, Q_{d}} \rightarrow \operatorname{Hilb}_{Q_{d}}
$$

is reduced.

Proof. Consider the natural projection map:

$$
\operatorname{pr}_{0}: \operatorname{Hilb}_{P_{0}, P_{1}, Q_{d}} \rightarrow \operatorname{Hilb}_{P_{0}}
$$

Clearly, this map is surjective as for every line $L$ there exists infinitely many lines $L^{\prime}$ lying on the same plane as $L$ such that $L \cup L^{\prime}$ is contained in a smooth, degree $d$ surface in $\mathbb{P}^{3}$. Note that for any point $t \in \operatorname{Hilb}_{P_{0}}$, we have

$$
\operatorname{dim} T_{t} \operatorname{Hilb}_{P_{0}}=h^{0}\left(\mathcal{N}_{L_{t} \mid \mathbb{P}^{3}}\right)=h^{0}\left(\mathcal{O}_{L_{t}}(1)\right)+h^{0}\left(\mathcal{O}_{L_{t}}(1)\right)=4=\operatorname{dim} \operatorname{Hilb}_{P_{0}},
$$

where $L_{t}$ is the line corresponding to the point $t$. Hence, Hilb $P_{P_{0}}$ is smooth. A standard exercise in commutative algebra tells us that given a morphism of schemes, the domain is reduced if the scheme-theoretic image and every fiber is reduced. Therefore, it is sufficient to check that the every fiber to the morphism $\mathrm{pr}_{0}$ is reduced.

Note that, the morphism $\operatorname{pr}_{0}$ factors through $\operatorname{Hilb}_{P_{0}, P_{1}}$. Denote by

$$
\mathrm{pr}_{1}: \operatorname{Hilb}_{P_{0}, P_{1}} \rightarrow \operatorname{Hilb}_{P_{0}} \text { and } \operatorname{pr}_{2}: \operatorname{Hilb}_{P_{0}, P_{1}, Q_{d}} \rightarrow \operatorname{Hilb}_{P_{0}, P_{1}}
$$

the natural projections. Since every conic can be embedded in a degree $d$ surface in $\mathbb{P}^{3}$, the scheme-theoretic image of $\mathrm{pr}_{0}^{-1}(t)$ under the morphism $\mathrm{pr}_{2}$ coincides with the fiber $\mathrm{pr}_{1}^{-1}(t)$. The dimension of $\operatorname{pr}_{1}^{-1}(t)$ equals $\operatorname{dim} \mathbb{P}\left(H^{0}\left(\mathcal{O}_{\mathbb{P}^{2}}(1)\right)\right)+1$, where the first term is the dimension of the space of lines contained in the same plane as $L_{t}$ (after fixing the plane) and the second term is the dimension of the space of planes in $\mathbb{P}^{3}$ containing $L_{t}$. For any line $L^{\prime}$ contained in the same plane as $L_{t}$, we have

$$
h^{0}\left(\mathcal{O}_{L_{t}+L^{\prime}}(1)\right)=h^{0}\left(\mathcal{O}_{L_{t}}(1)\right)+1 \text { and } h^{0}\left(\mathcal{O}_{L_{t}+L^{\prime}}(2)\right)=2.2+1=5
$$

where the last equality follows from the fact that the Castelnuovo-Mumford regularity of $\mathcal{O}_{L_{t}+L^{\prime}}$ is one, which implies $h^{0}\left(\mathcal{O}_{L_{t}+L^{\prime}}(2)\right)$ equals $P_{L_{t}+L^{\prime}}(2)$ for the Hilbert polynomial $P_{L_{t}+L^{\prime}}(n)$ of $L_{t}+L^{\prime}$. Similarly, $h^{0}\left(\mathcal{O}_{L_{t}}(2)\right)=2+1=3$. Since $\mathcal{N}_{L_{t}+L^{\prime} \mid \mathbb{P}^{3}} \cong \mathcal{O}_{L_{t}+L^{\prime}}(1) \oplus \mathcal{O}_{L_{t}+L^{\prime}}(2)$, [5, Ex. II.8.4] implies that the restriction morphism

$$
\rho: H^{0}\left(\mathcal{N}_{L_{t}+L^{\prime} \mid \mathbb{P}^{3}}\right) \rightarrow H^{0}\left(\mathcal{N}_{L_{t}+L^{\prime} \mid \mathbb{P}^{3}} \otimes_{\mathcal{O}^{3}} \mathcal{O}_{L_{t}}\right)
$$

is surjective. Hence,

$$
\operatorname{dim} \operatorname{ker}(\rho)=h^{0}\left(\mathcal{O}_{L_{t}+L^{\prime}}(1)\right)+h^{0}\left(\mathcal{O}_{L_{t}+L^{\prime}}(2)\right)-h^{0}\left(\mathcal{O}_{L_{t}}(1)\right)-h^{0}\left(\mathcal{O}_{L_{t}}(2)\right)=1+5-3=3 .
$$

Using [8, Remarks 4.5.4], we have $\operatorname{dim} T_{\left(L_{t} \subset L_{t}+L^{\prime}\right)} \operatorname{pr}_{1}^{-1}(t)=\operatorname{ker} \rho$, which by our computation equals dim $\mathrm{pr}_{1}^{-1}(t)$. Hence, $\mathrm{pr}_{1}^{-1}(t)$ is reduced.

The fiber over the point corresponding to the pair $\left(L_{t} \subset L_{t} \cup L^{\prime}\right)$ for the composed morphism

$$
\operatorname{pr}_{0}^{-1}(t) \hookrightarrow \operatorname{Hilb}_{P_{0}, P_{1}, Q_{d}} \stackrel{\mathrm{pr}_{2}}{\longrightarrow} \operatorname{Hilb}_{P_{0}, P_{1}}
$$

is isomorphic to $\mathbb{P}\left(H^{0}\left(\mathcal{I}_{L_{t} \cup L^{\prime}}(d)\right)\right)$, which is reduced. Since $\operatorname{pr}_{2}\left(\operatorname{pr}_{0}^{-1}(t)\right)=\operatorname{pr}_{1}^{-1}(t)$ is reduced, this implies that $\operatorname{pr}_{0}^{-1}(t)$ is reduced. Hence, Hilb $P_{0}, P_{1}, Q_{d}$ is reduced. The second part of the 
lemma is direct (scheme-theoretic image of a reduced scheme is reduced). This proves the proposition.

2.3. Proof of Theorem 1.1. Let $X$ be a general, smooth, degree $d$ surface in $\mathbb{P}^{3}$ containing 2 distinct coplanar lines, say $L_{1}, L_{2}$. We use the notations as in $\S 2.2$. Denote by $o \in U_{d}$ the point corresponding to $X$. Let $U \subset U_{d}$ be a simply connected neighbourhood of $o$ in $U_{d}$ as before.

Proposition 2.3. The (Zariski) closure of $\mathrm{NL}\left(\left[L_{1}\right]\right) \cap \mathrm{NL}\left(\left[L_{2}\right]\right)$ in $U_{d}$ is isomorphic to the scheme-theoretic image of the morphism pr as in Proposition 2.2, intersected with $U_{d}$.

Proof. Denote by $W:=\operatorname{Im}(\mathrm{pr}) \cap U_{d}$, where pr is as in Proposition 2.2. Note that, $W$ parametrizes smooth, degree $d$ surfaces in $\mathbb{P}^{3}$ containing two coplanar lines. Hence, $\overline{\mathrm{NL}\left(\left[L_{1}\right]\right) \cap \mathrm{NL}\left(\left[L_{2}\right]\right)}$ contains $W$. We now prove the reverse inclusion i.e., $\overline{\mathrm{NL}\left(\left[L_{1}\right]\right) \cap \mathrm{NL}\left(\left[L_{2}\right]\right)} \subset W$. Denote by

$$
\pi^{\prime}: \mathcal{X}^{\prime} \rightarrow \overline{\mathrm{NL}\left(\left[L_{1}\right]\right) \cap \mathrm{NL}\left(\left[L_{2}\right]\right)}
$$

the restriction of $\pi$ to $\overline{\mathrm{NL}\left(\left[L_{1}\right]\right) \cap \mathrm{NL}\left(\left[L_{2}\right]\right)}$. By Lefschetz $(1,1)$-theorem, there exist invertible sheaves $\mathcal{L}_{1}$ and $\mathcal{L}_{2}$ over $\mathcal{X}^{\prime}$ such that $\left.\mathcal{L}_{1}\right|_{X} \cong \mathcal{O}_{X}\left(L_{1}\right)$ and $\left.\mathcal{L}_{2}\right|_{X} \cong \mathcal{O}_{X}\left(L_{2}\right)$. Using Lemma 2.1 and the upper semi-continuity of cohomology (see [5, Theorem III.12.8]), there exists an open neighbourhood $V \subset \overline{\mathrm{NL}\left(\left[L_{1}\right]\right) \cap \mathrm{NL}\left(\left[L_{2}\right]\right)}$ of $o$ such that for all $v \in V$, we have

$$
h^{0}\left(\mathcal{L}_{1, v}\right)=1=h^{0}\left(\mathcal{L}_{2, v}\right) \text { and } h^{1}\left(\mathcal{L}_{1, v}\right)=0=h^{1}\left(\mathcal{L}_{2, v}\right) \text {, where } \mathcal{L}_{1, v}:=\mathcal{L}_{1} \mid \mathcal{X}_{v} \text { and } \mathcal{L}_{2, v}:=\mathcal{L}_{2} \mid \mathcal{X}_{v} .
$$

By [5, Theorem III.12.11], for every $v \in V$, the natural morphisms

$$
\pi_{*}^{\prime} \mathcal{L}_{1} \otimes k(v) \rightarrow H^{0}\left(\mathcal{L}_{1, v}\right) \text { and } \pi_{*}^{\prime} \mathcal{L}_{2} \otimes k(v) \rightarrow H^{0}\left(\mathcal{L}_{2, v}\right)
$$

are isomorphisms. Hence, after contracting $V$ if necessary, there exist sections $s_{1} \in \Gamma\left(V, \pi_{*}^{\prime} \mathcal{L}_{1}\right)$ and $s_{2} \in \Gamma\left(V, \pi_{*}^{\prime} \mathcal{L}_{2}\right)$ such that its image $s_{1, v}$ and $s_{2, v}$ in $H^{0}\left(\mathcal{L}_{1, v}\right)$ and $H^{0}\left(\mathcal{L}_{2, v}\right)$, respectively are non-zero for all $v \in V$. The sections $s_{1}$ and $s_{2}$ give rise to the short exact sequence:

$$
\left.0 \rightarrow \mathcal{L}_{i}^{\vee}\right|_{V} \stackrel{s_{i}}{\rightarrow} \mathcal{O}_{\mathcal{X}_{V}} \rightarrow \mathcal{O}_{Z\left(s_{i}\right)} \rightarrow 0
$$

where $Z\left(s_{i}\right)$ is the zero locus of the section $s_{i}$ in $\mathcal{X}_{V}:=\pi^{-1}(V)$, for $i=1,2$. Since $s_{i, v}$ is non-zero, the natural morphism

$$
\mathcal{L}_{i, v}^{\vee} \stackrel{\cdot s_{i, v}}{\longrightarrow} \mathcal{O}_{\mathcal{X}_{v}}
$$

is injective for $i=1,2$. By the local criterion of flatness (see [7, p. 150, (20.E)]), we conclude that $Z\left(s_{i}\right)$ is flat over $V$ for $i=1,2$. Denote by $\mathcal{L}_{1}^{\text {eff }}:=Z\left(s_{i}\right)$ and $\mathcal{L}_{2}^{\text {eff }}:=Z\left(s_{2}\right)$. It is easy to check that the effective divisor $\mathcal{L}_{1}^{\text {eff }}+\mathcal{L}_{2}^{\text {eff }}$ of $\mathcal{X}_{V}$ is also flat over $V$. By the universal property, of the flag Hilbert schemes (see [8, Theorem 4.5.1]), the triple

$$
\left(\mathcal{L}_{1}^{\text {eff }} \subset \mathcal{L}_{1}^{\text {eff }}+\mathcal{L}_{2}^{\text {eff }} \subset \mathcal{X}_{V}\right)
$$

induces a morphism from $V$ to $\operatorname{Hilb}_{P_{0}, P_{1}, Q_{d}}$ such that the composition

$$
V \rightarrow \operatorname{Hilb}_{P_{0}, P_{1}, Q_{d}} \stackrel{\mathrm{pr}}{\rightarrow} \operatorname{Hilb}_{Q_{d}}
$$

is the natural inclusion. This implies, a dense open subscheme of $\overline{\mathrm{NL}\left(\left[L_{1}\right]\right) \cap \mathrm{NL}\left(\left[L_{2}\right]\right)}$ lies in the scheme-theoretic image of pr. Since the morphism pr is proper, we conclude that $\overline{\mathrm{NL}}\left(\left[L_{1}\right]\right) \cap \mathrm{NL}\left(\left[L_{2}\right]\right)$ lies in the scheme-theoretic image of pr. So, we have the reverse inclusion. Hence. $\overline{\mathrm{NL}}\left(\left[L_{1}\right]\right) \cap \mathrm{NL}\left(\left[L_{2}\right]\right)=\operatorname{Im}(\mathrm{pr})$. This proves the proposition.

Theorem 2.4. For any $a \in \mathbb{Z}$, denote by $\gamma_{a}:=\left[L_{1}\right]+a\left[L_{2}\right] \in H^{2}(X, \mathbb{Z}) \cap H^{1,1}(X, \mathbb{C})$. Then, for any $a, b \geq 0$ with $a \neq b$, we have $\operatorname{NL}\left(\gamma_{a}\right) \neq \mathrm{NL}\left(\gamma_{b}\right)$. Moreover,

$\operatorname{codim} \mathrm{NL}\left(\gamma_{0}\right)=d-3, \operatorname{codim} \mathrm{NL}\left(\gamma_{1}\right)=2 d-7$ and $\operatorname{codim} \mathrm{NL}\left(\gamma_{a}\right)=2 d-6$ for $a \geq 2$. 
Proof. Denote by $\overline{\mathrm{NL}\left(\gamma_{a}\right)}$ the closure of $\mathrm{NL}\left(\gamma_{a}\right)$ in $U_{d}$ under the Zariski topology. By [9, Theorem $0.2] \overline{\mathrm{NL}\left(\gamma_{0}\right)}$ (resp. $\left.\overline{\mathrm{NL}\left(\gamma_{1}\right)}\right)$ parametrizes smooth, degree $d$ surfaces containing a line (resp. a conic) and is of codimension $d-3$ (resp. $2 d-7$ ) in $U_{d}$. Furthermore, both $\operatorname{NL}\left(\gamma_{0}\right)$ and $\operatorname{NL}\left(\gamma_{1}\right)$ are reduced. We first note that for $a \geq 2$, we have $\mathrm{NL}\left(\gamma_{a}\right) \neq \mathrm{NL}\left(\gamma_{0}\right), \operatorname{NL}\left(\gamma_{a}\right) \neq \mathrm{NL}\left(\gamma_{1}\right)$ and $\operatorname{codim} \mathrm{NL}\left(\gamma_{a}\right)=2 d-6$. Indeed, $\overline{\mathrm{NL}\left(\gamma_{a}\right)}$ contains the space $W$ parametrizing smooth, degree $d$ surfaces containing 2 coplanar lines. It is easy to compute that codim $W=2 d-6$. Hence, codim $\mathrm{NL}\left(\gamma_{a}\right) \leq 2 d-6$. By [9, Theorem 0.2], either $\mathrm{NL}\left(\gamma_{a}\right)=\mathrm{NL}\left(\gamma_{0}\right)$ or $\mathrm{NL}\left(\gamma_{a}\right)=\mathrm{NL}\left(\gamma_{1}\right)$ or $\operatorname{codim} \mathrm{NL}\left(\gamma_{a}\right)=2 d-6$. If $\mathrm{NL}\left(\gamma_{a}\right)=\mathrm{NL}\left(\gamma_{0}\right)$ or $\mathrm{NL}\left(\gamma_{a}\right)=\mathrm{NL}\left(\gamma_{1}\right)$, then $\overline{\mathrm{NL}\left(\gamma_{a}\right)}$ parametrizes smooth, degree $d$ surfaces such that both $\left[L_{1}\right]$ and $\left[L_{2}\right]$ remains a Hodge class, in particular

$$
\mathrm{NL}\left(\gamma_{a}\right)=\mathrm{NL}\left(\left[L_{1}\right]\right) \cap \mathrm{NL}\left(\left[L_{2}\right]\right) .
$$

But, Proposition 2.3 then implies that $\overline{\mathrm{NL}\left(\gamma_{a}\right)}=\operatorname{Im}(\mathrm{pr})=W$, which is of codimension $2 d-6$. This gives us a contradiction. Hence, for $a \geq 2, \mathrm{NL}\left(\gamma_{a}\right) \neq \mathrm{NL}\left(\gamma_{0}\right), \operatorname{NL}\left(\gamma_{a}\right) \neq \mathrm{NL}\left(\gamma_{1}\right)$ and $\operatorname{codim} \overline{\mathrm{NL}\left(\gamma_{a}\right)}=2 d-6$.

We now show that for $a \geq 2$ and $u \in \mathrm{NL}\left(\gamma_{a}\right)$ general, we have $\operatorname{codim} T_{u} \mathrm{NL}\left(\gamma_{a}\right) \leq 2 d-7$. Indeed, using [3, (4.a.4)], we have for all $a \geq 2$,

$$
H^{0}\left(K_{X}\left(-L_{1}-L_{2}\right)\right) \subset H^{2,0}\left(-\gamma_{a}\right):=\left\{\psi \in H^{2,0}(X, \mathbb{C}) \mid(t \cup \psi) \cup \gamma_{a}=0 \text { for all } t \in H^{1}\left(\mathcal{T}_{X}\right)\right\} .
$$

Recall from [3, p. 211] that

$$
\operatorname{codim} T_{o} \operatorname{NL}\left(\gamma_{a}\right)=\operatorname{dim} H^{2,0}(X, \mathbb{C})-\operatorname{dim} H^{2,0}\left(-\gamma_{a}\right)
$$

which by our calculations is bounded above by $h^{0}\left(K_{X}\right)-h^{0}\left(K_{X}\left(-L_{1}-L_{2}\right)\right)$. Consider now the short exact sequence:

$$
0 \rightarrow K_{X}\left(-L_{1}-L_{2}\right) \rightarrow K_{X} \rightarrow K_{X} \otimes \mathcal{O}_{L_{1} \cup L_{2}} \rightarrow 0 .
$$

Using [6, Example 1.8.2], the Castelnuovo-Mumford regularity of $\mathcal{O}_{L_{1} \cup L_{2}}$ is one. Hence,

$$
H^{1}\left(\mathcal{I}_{L_{1} \cup L_{2}}(d-4)\right)=0 \text { for } d \geq 6 .
$$

Using the exact sequence

$$
0 \rightarrow \mathcal{I}_{X}(d-4) \rightarrow \mathcal{I}_{L_{1} \cup L_{2}}(d-4) \rightarrow \mathcal{O}_{X}\left(-L_{1}-L_{2}\right)(d-4) \rightarrow 0
$$

we conclude that $H^{1}\left(\mathcal{O}_{X}\left(-L_{1}-L_{2}\right)(d-4)\right)=0$ for $d \geq 6$. In particular,

$$
H^{1}\left(K_{X}\left(-L_{1}-L_{2}\right)\right)=H^{1}\left(\mathcal{O}_{X}\left(-L_{1}-L_{2}\right)(d-4)\right)=0 .
$$

By the short exact sequence (2.4), we conclude that

$$
h^{0}\left(K_{X}\right)-h^{0}\left(K_{X}\left(-L_{1}-L_{2}\right)\right)=h^{0}\left(K_{X} \otimes \mathcal{O}_{L_{1} \cup L_{2}}\right)=h^{0}\left(\mathcal{O}_{L_{1} \cup L_{2}}(d-4)\right)=P_{L_{1} \cup L_{2}}(d-4),
$$

where $P_{L_{1} \cup L_{2}}(t)$ is the Hilbert polynomial of $L_{1} \cup L_{2}$ and the last equality follows from the fact that the Castelnuovo-Mumford regularity of $\mathcal{O}_{L_{1} \cup L_{2}}$ is one. Now, the Hilbert polynomial of $L_{1} \cup L_{2}$ is $2(d-4)+1=2 d-7$. Therefore, $\operatorname{codim} T_{o} \mathrm{NL}\left(\gamma_{a}\right) \leq 2 d-7$. Since $\mathrm{NL}\left(\left[L_{1}\right]\right) \cap \mathrm{NL}\left(\left[L_{2}\right]\right) \subset$ $\mathrm{NL}\left(\gamma_{a}\right)$ and both spaces are of the same dimension, we have $\mathrm{NL}\left(\left[L_{1}\right]\right) \cap \mathrm{NL}\left(\left[L_{2}\right]\right)=\mathrm{NL}\left(\gamma_{a}\right)_{\text {red }}$. Hence, for a general $u \in \mathrm{NL}\left(\gamma_{a}\right), L_{1}$ (resp. $L_{2}$ ) deforms to a line $L_{1, u}$ (resp. $L_{2, u}$ ) in $\mathcal{X}_{u}$ (use the construction of $\mathcal{L}_{1}^{\text {eff }}$ and $\mathcal{L}_{2}^{\text {eff }}$ from the proof of Proposition 2.3). Then, $\gamma_{a}$ deforms to $\gamma_{a, u}:=\left[L_{1, u}\right]+a\left[L_{2, u}\right]$. Hence, $\operatorname{NL}\left(\gamma_{a}\right)=\mathrm{NL}\left(\gamma_{a, u}\right)$. Similarly, as before, we get

$$
\operatorname{codim} T_{u} \mathrm{NL}\left(\gamma_{a}\right)=\operatorname{codim} T_{u} \mathrm{NL}\left(\gamma_{a, u}\right) \leq 2 d-7 .
$$

This proves our claim.

If for $a, a^{\prime} \geq 2$ with $a \neq a^{\prime}$, we have $\operatorname{NL}\left(\gamma_{a}\right)=\mathrm{NL}\left(\gamma_{a^{\prime}}\right)$, then clearly

$$
\overline{\mathrm{NL}\left(\gamma_{a}\right)}=\overline{\mathrm{NL}\left(\gamma_{a^{\prime}}\right)} \subset \overline{\mathrm{NL}\left(\left[L_{1}\right]\right) \cap \mathrm{NL}\left(\left[L_{2}\right]\right)} \text {. }
$$

Since the three spaces have the same dimension and $\overline{\mathrm{NL}\left(\left[L_{1}\right]\right) \cap \mathrm{NL}\left(\left[L_{2}\right]\right)}$ is reduced (use Proposition 2.2 combined with Proposition 2.3), they coincide. But, $\overline{\mathrm{NL}\left(\left[L_{1}\right]\right) \cap \mathrm{NL}\left(\left[L_{2}\right]\right)}$ is reduced 
and $\mathrm{NL}\left(\gamma_{a}\right), \mathrm{NL}\left(\gamma_{a^{\prime}}\right)$ are generically non-reduced, as observed earlier. This gives a contradiction, hence $\mathrm{NL}\left(\gamma_{a}\right) \neq \mathrm{NL}\left(\gamma_{a^{\prime}}\right)$.

Remark 2.5. After Theorem 2.4, the remaining parts of Theorem 1.1 follows directly from [9, Theorem 0.2].

\section{REFERENCES}

[1] C. Ciliberto, J. Harris, and R. Miranda. General components of the Noether-Lefschetz locus and their density in the space of all surfaces. Mathematische Annalen, 282(4):667-680, 1988.

[2] M. Green. Components of maximal dimension in the Noether-Lefschetz locus. J. Differential Geometry, 29:295-302, 1989 .

[3] P. Griffiths and J. Harris. Infinitesimal variations of Hodge structure (II): an infinitesimal invariant of Hodge classes. Composition Mathematica, 50(2-3):207-265, 1983

[4] Movasati H. Special components of Noether-Lefschetz locus. arXiv preprint arXiv:1908.04117, 2019.

[5] R. Hartshorne. Algebraic Geometry. Graduate text in Mathematics-52. Springer-Verlag, 1977.

[6] R. K. Lazarsfeld. Positivity in algebraic geometry I: Classical setting: line bundles and linear series, volume 48. Springer, 2017.

[7] H. Matsumura. Commutative ring theory, volume 8. Cambridge university press, 1989.

[8] E. Sernesi. Deformaions of Algebraic Schemes. Grundlehren der Mathematischen Wissenschaften-334. Springer-Verlag, 2006.

[9] C. Voisin. Composantes de petite codimension du lieu de Noether-Lefschetz. Comm. Math. Helve., 64(4):515$526,1989$.

[10] C. Voisin. Sur le lieu de Noether-Lefschetz en degrés 6 et 7. Compositio Mathematica, 75(1):47-68, 1990.

11] C. Voisin. Contrexemple à une conjecture de J. Harris. Comptes rendus de l'Académie des sciences. Série 1, Mathématique, 313(10):685-687, 1991.

[12] C. Voisin. Hodge Theory and Complex Algebraic Geometry-I. Cambridge studies in advanced mathematics-76. Cambridge University press, 2002.

[13] C. Voisin. Hodge Theory and Complex Algebraic Geometry-II. Cambridge studies in advanced mathematics77. Cambridge University press, 2003.

BCAM - Basque Centre for Applied Mathematics, Alameda de Mazarredo 14, 48009 Bilbao, SPAIN

Email address: adan@bcamath.org 